\title{
The role of infection in the pathogenesis of idiopathic pulmonary fibrosis
}

\author{
Philip L. Molyneaux ${ }^{1,2}$ and Toby M. Maher ${ }^{1,2,3}$
}

Affiliations: 'Dept of Respiratory Medicine, Interstitial Lung Disease Unit, Royal Brompton Hospital, London, UK. ${ }^{2}$ National Heart and Lung Institute, Imperial College London, London, and ${ }^{3}$ Centre for Respiratory Research, University College London, London, UK.

Correspondence: T.M. Maher, Interstitial Lung Disease Unit, Royal Brompton Hospital, Sydney Street, London, SW3 6NP, UK. E-mail: t.maherđrbht.nhs.uk

ABSTRACT Idiopathic pulmonary fibrosis (IPF) is a progressive, and invariably fatal, condition that is believed to arise in genetically susceptible individuals as a consequence of an aberrant wound-healing response following repetitive alveolar injury. The exact triggers, which initiate the fibrotic process, remain unknown. Infectious agents, including both viruses and bacteria, have the capacity to cause alveolarepithelial cell injury and apoptosis. Relatively few studies have examined the role of infection in IPF. Those that have, point to viruses playing a key role as cofactors in the initiation and progression of IPF. There is also some evidence to suggest that viral infection may be responsible for a proportion of acute exacerbations of IPF. The role played by bacteria in the pathogenesis of IPF is less clear cut. Studies from other respiratory diseases suggest that alterations in the lung microbiome are associated with disease and that these changes influence disease behaviour. Emerging molecular microbiological techniques are making the study of microbial communities in the lung easier. It is hoped that by combining such techniques with the careful longitudinal phenotyping of patients with IPF, it will be possible to elucidate the role played by bacteria and viruses in the pathogenesis of the disease. If infection plays a causal role in IPF then it is possible that therapeutic strategies, utilising currently available antiviral or antibiotic drugs, may be effective in modifying the course of this devastating condition.

0 @ERSpublications

Understanding viral and bacterial involvement in IPF could help development of novel therapeutic approaches http://ow.ly/mMIMg

\section{Introduction}

While the pathogenesis of idiopathic pulmonary fibrosis (IPF) remains poorly understood the condition is thought to result from repeated episodes of alveolar injury in individuals with dysfunctional alveolar wound-healing mechanisms [1]. This paradigm suggests a role in IPF for both environmental and host factors, with, in all likelihood, interactions between the two. Recent studies have contributed to a growing understanding of the complex genetics underlying IPF $[2,3]$. However, despite epidemiological studies highlighting several environmental exposures, which confer an increased risk for developing IPF [4], the unifying trigger (or triggers), which initiate the fibrotic process in genetically susceptible individuals, remain unknown. The temporal and spatial heterogeneity observed in usual interstitial pneumonia (the histological correlate of IPF) speaks to the likely importance of repetitive injury as a key initiating factor in the pathogenesis of the disease. Of all the possible factors giving rise to alveolar injury, including dusts, pollution, fibres (particularly asbestos), and gastric aspirate, probably the most commonly encountered but least appreciated, in the context of fibrosis, is infection. Recent studies have pointed to disordered host defence and thus susceptibility to infection, as an important contributor to disease progression in IPF.

Received: Feb 242013 | Accepted after revision: April 042013

Conflict of interest: None declared.

Provenance: Submitted article, peer reviewed.

Copyright @ERS 2013 
The PANTHER-IPF (Prednisone, Azathioprine, and N-acetylcysteine: A Study That Evaluates Response in IPF) study has clearly shown that immunosuppression with corticosteroids and azathioprine is deleterious in individuals with IPF $[5,6]$. Meanwhile, analysis of the peripheral blood transcriptome has highlighted deficiencies in a number of genes related to host defence in IPF [7]. This review therefore seeks to redress the balance and describes current knowledge of the role played by infection in the pathogenesis, progression and (acute) exacerbation of IPF.

\section{Infection and the pathogenesis of IPF}

Viruses have long been suspected of playing a role in the pathogenesis of IPF (table 1), and it is well recognised that many patients report a viral-type prodrome preceding the initial development of respiratory symptoms [24]. However, the evidence supporting a role for viruses in IPF is scant and often conflicting. Hepatitis $\mathrm{C}(\mathrm{HCV})$ is a positive-sense RNA virus that frequently causes fibrosis in the liver resulting in cirrhosis. A number of case reports and case series have suggested a role for HCV in the development of IPF. In a Japanese study, which contained 66 subjects with IPF and 9646 controls, UEDA et al [8] found that $28.8 \%$ of IPF subjects, but only $3.66 \%$ of controls, had evidence of prior HCV infection. ARASE et al. [9] addressed the same question from the opposite direction. They studied a cohort of 6150 patients with HCV and a cohort of 2050 patients with Hepatitis B (HBV). In the group with HCV they noted a 10-year and 20year cumulative incidence of IPF of $0.3 \%$ and $0.9 \%$, respectively. This compared to no cases of IPF in the HBV group. Multivariate analysis suggested that advancing age, smoking and liver cirrhosis were all independent risk factors for the development of IPF. However, a number of studies have failed to replicate this association [10] and some have simply demonstrated an association of HCV with a range of nonfibrotic respiratory conditions [11]. These differences between studies may reflect geographical variations in the incidence of HCV. The lack of a coherent signal across studies suggests that HCV is unlikely to be an important trigger for the development of IPF.

In addition to systemic infection, the lung is frequently exposed to airborne viruses and there is growing evidence, obtained both from human tissue and animal models, to support a mechanistic role for these pathogens in the initiation and progression of IPF. The human herpes viruses (HHVs) are a large family of ubiquitous DNA viruses that include the common pathogens herpes simplex virus type 1 (HSV-1), Epstein-Barr virus (EBV), cytomegalovirus (CMV) and HHV-7 and -8. VERGNON et al. [14], in the first study to suggest an association between HHV and IPF, reported that 12 out of 13 IPF patients were seropositive for EBV compared to none of the 12 patients with other forms of interstitial lung disease. Several other studies have reported similar findings of an increased incidence of EBV in lung biopsy and bronchoalveolar lavage (BAL) specimens from IPF

TABLE 1 A synopsis of studies linking viruses with the pathogenesis, progression and lacute) exacerbation of idiopathic pulmonary fibrosis (IPF)

\section{Virus \\ Major study findings \\ Comment}

HCV $28 \%$ of an IPF cohort had evidence of prior HCV compared with $3.6 \%$ of controls [8]

Incidence of IPF higher in cohort of HCV positive individuals compared to cohort with HBV [9]

No association between IPF and HCV [10]

Association of $\mathrm{HCV}$ with a range of non-fibrotic respiratory conditions [11]

TTV Lower survival rate in IPF patients with presence of TTV-DNA in serum compared to IPF with no TTV-DNA [12]

Detected in BAL during IPF exacerbation and in individuals with acute lung injury [13]

HHV 12 out of 13 IPF patients seropositive for EBV [14]

Increased EBV in lung biopsy samples and BAL from individuals with IPF compared with controls [15-17]

At least one HHV detected in $97 \%$ of IPF patients compared with $36 \%$ controls $[18,19]$.

MHV triggers and enhances fibrotic response in mice [20-23]
The lack of a coherent signal across studies suggests that $\mathrm{HCV}$ is unlikely to be an important trigger for the development of IPF

Link to pathogenesis unclear.

May cause progression of disease or be linked to the development of acute exacerbations

Some conflicting evidence but potential role of $\mathrm{HHVs}$ as a co-factor in the initiation and progression of IPF

HCV: hepatitis C virus; TTV: transfusion transmitted virus; HHV: human herpes virus; HBV: hepatitis B virus; BAL: bronchoalveolar lavage; EBV: Epstein-Barr virus; MHV: murine herpes virus. 
subjects when compared to controls $[15-17,25]$. Whilst EBV has been the most frequently studied of the HHVs, TANG et al. [18] looked more broadly at a range of HHV family members and found evidence of past infection with at least one HHV in $97 \%$ of patients with IPF compared to only $36 \%$ of healthy controls. Over $50 \%$ of the IPF patients in their study had evidence of past infection with at least two HHVs. Molecular techniques undertaken in IPF lung tissue suggest the presence of latent, lytic HHVs, within the alveolar epithelium. It has been suggested that reactivation of this virus acts as a second hit to the epithelium following exposure to a first injurious insult [26]. Whilst this is an attractive hypothesis it should be emphasised that all the studies to date have been retrospective in design and have been undertaken in very small cohorts. Furthermore, these studies only demonstrate an association of HHV with IPF, rather than a causal relationship.

Given the limitations of the in vivo human studies conducted thus far, it is instructive to look at what has been learnt from animal models of fibrosis. Murine herpes virus type 68 (MHV-68) is closely related to HHV and, like its human counterpart, infects respiratory epithelium [27]. Infecting young, healthy mice with MHV-68 does not result in pulmonary fibrosis. However, when MHV-68 is administered in conjunction with known fibrotic stimuli (e.g. bleomycin or fluorescein isothiocyanate (FITC)) it dramatically enhances lung fibrosis $[20,21]$. This observation gives credence to the suggestion that viral infection may be an important cofactor in the development of IPF. Interestingly, and in further support of the human data, mice latently infected with MHV-68 prior to a fibrotic challenge (with either bleomycin or FITC) developed an exaggerated fibrotic response compared to non-infected animals [22]. This increase in fibrosis occurs without any evidence of reactivation of MHV-68. Other observations in mice that may be relevant to IPF include the finding that interferon- $\gamma$ deficient mice (animals that mimic the T-helper cell type 2 predominant cytokine environment found in the lungs of patients with IPF) develop fibrosis when infected with MHV-68 alone [21]. Similarly, senescent, but not young, mice (aged between 15-18 months, equivalent to human age of 60-75 years) also develop pulmonary fibrosis when infected with MHV-68 [23].

\section{Viruses and IPF disease mechanisms}

The mechanisms by which viruses might predispose to the development of IPF are beginning to be elucidated. HHV induces endoplasmic reticulum (ER) stress and apoptosis in vitro in epithelial cells [28]. In vivo, in human IPF biopsy samples, LAWSON et al. [29] have shown co-localisation of latent HHV and markers of ER stress and apoptosis [29]. Activation of these pathways has been highlighted in the development of both IPF and the pulmonary fibrosis associated with the rare genetic disorder HumanskyPudlak syndrome [30, 31]. In the murine bleomycin model, chronic MHV-68 infection results in deposition of collagen, increased tumour growth factor (TGF)- $\beta$ expression and the altered synthesis of surfactant proteins [22]. Similarly, MHV-68-induced pulmonary fibrosis in aged mice is associated with up-regulation of the potent pro-fibrotic growth factor, TGF- $\beta$ [23].

If viral infection is important in either the development or progression of IPF then it might be hoped that effective antiviral therapy will have a disease modifying effect in IPF. Stabilisation of IPF, through the administration of antiviral therapy, has been described in case reports, albeit in individuals with evidence of infection on either BAL or biopsy [18]. More recently EGAN et al. [32] have reported a small study of openlabel ganciclovir in 14 subjects with severe IPF and positive EBV-IgG serology. Following a 2-week course of ganciclovir, nine of the 14 subjects showed improvements at 8 weeks in at least three of the four measured composite outcome. These included the reduction in steroid dose, the change in forced vital capacity, and an improvement in DTPA (diethylenetriaminepentaacetic acid) clearance. The small study size, the openlabelled design, the short duration of the study, and the lack of a control arm, all contribute in making it impossible to draw any conclusions concerning the efficacy of antiviral therapy as a means of stabilising IPF. Nonetheless, the study is provocative and provides a rationale for larger future trials of antiviral treatment in IPF.

\section{Infection and acute exacerbations of IPF}

Acute exacerbations are devastating episodes of unheralded, rapidly-progressive, respiratory compromise that occur in individuals with IPF [33]. Histologically, acute exacerbations are characterised by the finding of diffuse alveolar damage (DAD). Such episodes have been shown in clinical trials to affect between 4-15\% of individuals with IPF per year, and are an important cause of IPF-related mortality with a 3-month survival of $<50 \%[34,35]$. Current international diagnostic guidelines emphasise that acute exacerbations are cryptogenic and can only be confirmed following the exclusion of infection. A number of insults have been shown to trigger the development of DAD in IPF patients (where a cause is identified the current nomenclature precludes such episodes being defined as acute exacerbations), in particular thoracic surgery and drugs. In individuals with IPF, respiratory tract infections that necessitate hospitalisation confer a mortality risk indistinguishable from that seen with acute exacerbations [36]. Post mortem examination in such cases frequently discloses associated DAD. Given the lack of sensitivity encountered with current 
culture-dependant clinical microbiology techniques and the limited repertoire of tools available to diagnose acute viral infections, it is plausible to hypothesise that many episodes of apparent acute exacerbations simply represent the sequelae of infection. So is their evidence to support this notion?

In animal models there is now good evidence to show that viral infection can exacerbate established fibrosis and in so doing gives rise to a lesion resembling DAD [37]. However, in a study of 43 individuals suffering an acute exacerbation of IPF, WоOTTON et al. [13] failed to clearly identify a viral or other infectious trigger for the acute exacerbation in the vast majority of their subjects. All 43 of the acute exacerbations of IPF subjects had negative bacterial cultures and negative viral serology. Subsequent PCR analysis of BAL fluid (BALF) identified four samples positive for rhinovirus, parainfluenza or coronavirus. However, there was no evidence of detectable viruses on PCR in the BALF fluid from 40, stable, IPF patients $(\mathrm{p}=0.12)$. In addition to PCR, WOоTTON et al. [13] went on to employ pan-viral microarrays, which identified additional evidence of HSV, EBV and, interestingly, 12 cases of transfusion transmitted virus (TTV) in the exacerbation cohort with no viruses detected in the stable disease group. Deep sequencing of samples was also used to look for novel viruses not detectable by PCR or array based methods, but this did not identify any additional viruses. Although TTV was not found in stable IPF patients, it was found with a similar incidence in a cohort of controls with acute lung injury. The authors, therefore, conclude that their findings may simply reflect the sequelae of severe underlying inflammation rather than proving a link between viral infection and acute exacerbations of IPF. Similarly a study of the gene transcription profile of the lungs taken from eight patients who died from an acute exacerbation of IPF failed to identify a signature that might be expected in viral infection [38]. However, unlike other respiratory conditions where exacerbations are truly acute events, the onset of an acute exacerbation in IPF is generally more insidious [39]. It is possible, therefore, that by the time of presentation any triggering viruses would no longer be detectable.

\section{The role of bacteria in the pathogenesis of IPF}

Whilst there is some evidence to suggest a role for viruses in the pathogenesis of IPF, any role of bacteria is much less well established. The only observational evidence comes from RicHTER et al. [40], who in 2008 demonstrated positive BAL cultures (for known pathogens including Haemophilus, Streptococcus and Pseudomonas) in eight of 22 stable IPF patients. More recently a large multicentre, randomised, placebocontrolled study evaluated the prophylactic use of 12 months of septrin as a treatment for IPF [41]. The authors reported that there was no difference in the primary end-point of change in vital capacity when comparing septrin and placebo. Whilst there were a large number of drop-outs in the septrin arm of the study, post hoc analysis suggested that, in treatment adherent subjects, septrin led to a reduction in infections and mortality. This observation, together with the high mortality associated with bacterial respiratory tract infection in IPF, suggests that bacteria may play a role in driving IPF disease progression.

This lack of interest in the role of bacteria in the pathogenesis of IPF is predominantly because of the longstanding, but incorrect, belief that the lower airways are sterile. Recently, molecular cultureindependent techniques have identified complex microbial communities in the lower airways with distinct alterations in the microbiome occurring in a number of respiratory conditions [42-44]. Molecular techniques enable a full visualisation of the microbiota in any given environment. These techniques have yet to be fully applied to IPF. A small study, principally looking at the potential role of chronic Pneumocystis jirovecii infection in IPF, demonstrated a number of uncultured bacteria in the BAL of IPF patients, using basic, culture-independent techniques [45]. The application of molecular techniques to the field of bacteriology has been throwing up exciting insights in to disease pathogenesis in other organ systems [46]. To date, there has been no assessment of the role of the respiratory microbiome in the pathogenesis, progression or exacerbation of IPF. However, it is anticipated that the assessment of the lung microbiome in IPF will shed important light on the role played by bacterial colonisation of the lower airways in the development and progression of fibrosis [47].

\section{Conclusions}

Environmental triggers are almost certainly integral to the pathogenesis and progression of IPF. Both viruses and bacteria have the potential to cause airway epithelial cell injury and apoptosis and both have the capacity to modulate the host response to injury. Whilst active infection in IPF is known to carry a high morbidity and mortality, the effect of latent viral infection or changes in the composition of the lung microbiome remains unknown. Modern microbiological techniques permit a detailed analysis of lung pathogens. As such, it is to be hoped that the application of these methodologies to large populations, of greatly phenotyped IPF individuals, will help define the role played by viruses and bacteria in the development, progression and acute exacerbations in IPF patients. This should, in turn, enable the development of novel therapeutic approaches for this devastating disease. 


\section{References}

1 Maher TM, Wells AU, Laurent GJ. Idiopathic pulmonary fibrosis: multiple causes and multiple mechanisms? Eur Respir J 2007; 30: 835-839.

2 Seibold MA, Wise AL, Speer MC, et al. A common MUC5B promoter polymorphism and pulmonary fibrosis. N Engl J Med 2011; 364: 1503-1512.

3 du Bois RM. An earlier and more confident diagnosis of idiopathic pulmonary fibrosis. Eur Respir Rev 2012; 21: $141-146$.

4 Taskar VS, Coultas DB. Is idiopathic pulmonary fibrosis an environmental disease? Proc Am Thorac Soc 2006; 3: 293-298.

5 Idiopathic Pulmonary Fibrosis Clinical Research Network. Prednisone, azathioprine, and N-acetylcysteine for pulmonary fibrosis. N Engl J Med 2012; 366: 1968-1977.

6 Richeldi L. Assessing the treatment effect from multiple trials in idiopathic pulmonary fibrosis. Eur Respir Rev 2012; 21: 147-151.

$7 \quad$ Yang IV, Luna LG, Cotter J, et al. The peripheral blood transcriptome identifies the presence and extent of disease in idiopathic pulmonary fibrosis. PloS One 2012; 7: e37708.

8 Ueda T, Ohta K, Suzuki N, et al. Idiopathic pulmonary fibrosis and high prevalence of serum antibodies to hepatitis C virus. Am Rev Respir Dis 1992; 146: 266-268.

9 Arase Y, Suzuki F, Suzuki Y, et al. Hepatitis C virus enhances incidence of idiopathic pulmonary fibrosis. World J Gastroenterol 2008; 14: 5880-5886.

10 Irving WL, Day S, Johnston ID. Idiopathic pulmonary fibrosis and hepatitis C virus infection. Am Rev Respir Dis 1993; 148: 1683-1684.

11 Meliconi R, Andreone P, Fasano L, et al. Incidence of hepatitis C virus infection in Italian patients with idiopathic pulmonary fibrosis. Thorax 1996; 51: 315-317.

12 Bando M, Ohno S, Oshikawa K, et al. Infection of TT virus in patients with idiopathic pulmonary fibrosis. Respir Med 2001; 95: 935-942.

13 Wootton SC, Kim DS, Kondoh Y, et al. Viral infection in acute exacerbation of idiopathic pulmonary fibrosis. Am J Respir Crit Care Med 2011; 183: 1698-1702.

14 Vergnon JM, Vincent M, de Thé G, et al. Cryptogenic fibrosing alveolitis and Epstein-Barr virus: an association? Lancet 1984; 2: 768-771.

15 Manika K, Alexiou-Daniel S, Papakosta D, et al. Epstein-Barr virus DNA in bronchoalveolar lavage fluid from patients with idiopathic pulmonary fibrosis. Sarcoidosis Vasc Diffuse Lung Dis 2007; 24: 134-140.

16 Stewart JP, Egan JJ, Ross AJ, et al. The detection of Epstein-Barr virus DNA in lung tissue from patients with idiopathic pulmonary fibrosis. Am J Respir Crit Care Med 1999; 159: 1336-1341.

17 Kelly BG, Lok SS, Hasleton PS, et al. A rearranged form of Epstein-Barr virus DNA is associated with idiopathic pulmonary fibrosis. Am J Respir Crit Care Med 2002; 166: 510-513.

18 Tang YW, Johnson JE, Browning PJ, et al. Herpesvirus DNA is consistently detected in lungs of patients with idiopathic pulmonary fibrosis. J Clin Microbiol 2003; 41: 2633-2640.

19 Lasithiotaki I, Antoniou KM, Vlahava VM, et al. Detection of herpes simplex virus type-1 in patients with fibrotic lung diseases. PloS One 2011; 6: e27800.

20 Lok SS, Haider Y, Howell D, et al. Murine gammaherpes virus as a cofactor in the development of pulmonary fibrosis in bleomycin resistant mice. Eur Respir J 2002; 20: 1228-1232.

21 Mora AL, Woods CR, Garcia A, et al. Lung infection with $\gamma$ herpesvirus induces progressive pulmonary fibrosis in Th2-biased mice. Am J Physiol Lung Cell Mol Physiol 2005; 289: L711-L721.

22 Stoolman JS, Vannella KM, Coomes SM, et al. Latent infection by $\gamma$ herpesvirus stimulates profibrotic mediator release from multiple cell types. Am J Physiol Lung Cell Mol Physiol 2011; 300: L274-L285.

23 Naik PN, Horowitz JC, Moore TA, et al. Pulmonary fibrosis induced by gamma-herpesvirus in aged mice is associated with increased fibroblast responsiveness to transforming growth factor-beta. J Gerontol A Biol Sci Med Sci 2012; 67: 714-725.

24 Golden A, Bronk TT. Diffuse interstitial fibrosis of lungs; a form of diffuse interstitial angiosis and reticulosis of the lungs. AMA Arch Intern Med 1953; 92: 106-114.

25 Calabrese F, Kipar A, Lunardi F, et al. Herpes virus infection is associated with vascular remodeling and pulmonary hypertension in idiopathic pulmonary fibrosis. PloS One 2013; 8: e55715.

26 Kropski JA, Lawson WE, Blackwell TS. Right place, right time: the evolving role of herpesvirus infection as a "second hit" in idiopathic pulmonary fibrosis. Am J Physiol Lung Cell Mol Physiol 2012; 302: L441-L444.

27 Barton E, Mandal P, Speck SH. Pathogenesis and host control of gammaherpesviruses: lessons from the mouse. Annu Rev Immunol 2011; 29: 351-397.

28 Isler JA, Skalet AH, Alwine JC. Human cytomegalovirus infection activates and regulates the unfolded protein response. J Virol 2005; 79: 6890-6899.

29 Lawson WE, Crossno PF, Polosukhin VV, et al. Endoplasmic reticulum stress in alveolar epithelial cells is prominent in IPF: association with altered surfactant protein processing and herpesvirus infection. Am J Physiol Lung Cell Mol Physiol 2008; 294: L1119-L1126.

30 Young LR, Pasula R, Gulleman PM, et al. Susceptibility of Hermansky-Pudlak mice to bleomycin-induced type II cell apoptosis and fibrosis. Am J Respir Cell Mol Biol 2007; 37: 67-74.

31 Maher TM, Evans IC, Bottoms SE, et al. Diminished prostaglandin E2 contributes to the apoptosis paradox in idiopathic pulmonary fibrosis. Am J Respir Crit Care Med 2010; 182: 73-82.

32 Egan JJ, Adamali HI, Lok SS, et al. Ganciclovir antiviral therapy in advanced idiopathic pulmonary fibrosis: an open pilot study. Pulm Med 2011; 2011: 240805.

33 Collard HR, Moore BB, Flaherty KR, et al. Acute exacerbations of idiopathic pulmonary fibrosis. Am J Respir Crit Care Med 2007; 176: 636-643.

34 Judge EP, Fabre A, Adamali HI, et al. Acute exacerbations and pulmonary hypertension in advanced idiopathic pulmonary fibrosis. Eur Respir J 2012; 40: 93-100.

35 Mura M, Porretta MA, Bargagli E, et al. Predicting survival in newly diagnosed idiopathic pulmonary fibrosis: a 3year prospective study. Eur Respir J 2012; 40: 101-109. 

lung disease: aetiology and outcomes. Respirology 2010; 15: 909-917.

37 McMillan TR, Moore BB, Weinberg JB, et al. Exacerbation of establishec gammaherpesvirus. Am J Respir Crit Care Med 2008; 177: 771-780.

38 Konishi K, Gibson KF, Lindell KO, et al. Gene expression profiles of acute exacerbations of idiopathic pulmonary fibrosis. Am J Respir Crit Care Med 2009; 180: 167-175.

39 Cottin V. Changing the idiopathic pulmonary fibrosis treatment approach and improving patient outcomes. Eur Respir Rev 2012; 21: 161-167.

40 Richter AG, Stockley RA, Harper L, et al. Pulmonary infection in Wegener granulomatosis and idiopathic pulmonary fibrosis. Thorax 2009; 64: 692-697.

41 Shulgina L, Cahn AP, Chilvers ER, et al. Treating idiopathic pulmonary fibrosis with the addition of cotrimoxazole: a randomised controlled trial. Thorax 2013; 68: 155-162.

42 Beasley V, Joshi PV, Singanayagam A, et al. Lung microbiology and exacerbations in COPD. Int J Chron Obstruct Pulm Dis 2012; 7: 555-569.

43 Hilty M, Burke C, Pedro H, et al. Disordered microbial communities in asthmatic airways. PloS One 2010 ; 5: e8578.

44 Huang YJ, Kim E, Cox MJ, et al. A persistent and diverse airway microbiota present during chronic obstructive pulmonary disease exacerbations. OMICS 2010; 14: 9-59.

45 Friaza V, la Horra C, Rodríguez-Domínguez MJ, et al. Metagenomic analysis of bronchoalveolar lavage samples from patients with idiopathic interstitial pneumonia and its antagonic relation with Pneumocystis jirovecii colonization. J Microbiol Methods 2010; 82: 98-101.

46 Human Microbiome Project Consortium. Structure, function and diversity of the healthy human microbiome Nature 2012; 486: 207-214

47 Molyneaux PL, Russell AM, Cox MJ, et al. The respiratory microbiome in idiopathic pulmonary fibrosis. Am J Respir Crit Care Med 2012; 185: A5174. 